\section{FRI0221 GIANT CELL ARTERITIS PRESENTING AS FEVER OF UNKNOWN ORIGIN AND DELAY IN DIAGNOSIS: ANALYSIS OF TWO DIFFERENT DECADES}

R. Talarico ${ }^{1}$, C. Stagnaro ${ }^{1}$, F. Ferro ${ }^{1}$, A. Figliomeni ${ }^{1}$, L. Carli ${ }^{1}$, M. Mosca ${ }^{1}$ on behalf of $\mathrm{n} / \mathrm{a} .{ }^{1}$ Rheumatology Unit, Department of Clinical and Experimental Medicine, University of Pisa, Pisa, Italy

Background: Giant cell arteritis (GCA) represents the most common primary vasculitis of the elderly, that usually involves large and medium sized arteries. The wide spectrum of clinical manifestations can extensively vary, from cranial symptoms, such as headache, jaw claudication or visual alterations, to constitutional symptoms, like fever, weight loss or asthenia. Fever of unknown origin (FUO) may sometimes represents the initial symptom of GCA and when it is not associated with other typical GCA features, the diagnosis can be unluckily delayed.

Objectives: The primary aim of the study was to identify the prevalence of GCA patients presenting as FUO. The secondary aims were to identify the delays in the diagnosis and to compare them between the last two decades.

Methods: Epidemiological and clinical data of 274 GCA patients followed in the last 20 years in our Unit were analysed. We quantified the latency period between the onset of signs and symptoms and the final diagnosis of GCA in terms of months. Results: One hundred and eighty-five patients (49 males and 136 females, mean \pm SD age at the onset $71 \pm 7$ years) had shown at the onset signs and symptoms suggestive of GCA (new onset headache and/or scalp pain $86 \%$, jaw claudication $39 \%$, vision loss $35 \%$, abnormal temporal artery on examination $49 \%$, dizziness $31 \%$ ) while 89 patients ( 33 males and 56 females, mean age at the onset $69_{ \pm} 4$ years) were sent to our attention just for the onset of FUO and for an increase of erythrocyte sedimentation rate and C-reactive protein not otherwise justified. After an extensive work-up aimed at excluding any kind of infection, malignancy or hematological disorder, the patients with FUO performed a temporal artery biopsy (TAB) and/or a (18)F-fluorodeoxyglucose positron emission tomography (18F-FDG PET). The results from histology and/or imaging allowed us to perform the diagnosis of GCA in all cases; moreover the main PET alterations reported were characterized by a (18)FDG uptake of the aortic arch and its major braches, including the carotid, subclavian, thoracic aorta and, less frequently, the abdominal aorta. Considering the different decades, the mean latency period between the onset of FUO and the diagnosis of GCA was $6 \pm 3$ months in the decade from 2000 to 2010 and $3 \pm 2$ months in the last decade, that was significantly higher compared with the mean latency period between the onset of signs and symptoms suggestive of GCA and the definitive diagnosis ( $3 \pm 1$ months) in the other patients of the cohort in the first decade. Notably the latency period between the onset of signs and symptoms suggestive of GCA and the definitive diagnosis was more close $(2 \pm 1$ months) to the latency period of diagnosis in FUO presenting GCA in the last decade.

Conclusion: Our data underline that there is a major focus on the diagnosis of GCA, even when the presentation is not typical; this is probably due to the major knowledge reached in the last decade, to an improved sensibilization regarding the different profiles of presentation and surely on the bigger use of $18 \mathrm{~F}-\mathrm{FDG}$ PET in the work-up of GCA patients.

Acknowledgments: none

Disclosure of Interests: None declared

DOI: 10.1136/annrheumdis-2020-eular.5040

\section{FRI0222 MAGNETIC RESONANCE IMAGING OF MUSCLE INVOLVEMENT IN POLYARTERITIS NODOSA}

R. D. Vargas Ruíz ${ }^{1}$, J. A. Gómez Rosero ${ }^{1}$, C. Muñoz ${ }^{1,2,3}$, D. Jaramillo Arroyave $^{1,2,3,4}$, L. Hernandez $z^{1,5}$, L. A. González ${ }^{1}$, G. Vásquez ${ }^{1}$, M. Restrepo Escobar $^{1}$, A. Vanegas ${ }^{1,2} .{ }^{1}$ Universidad de Antioquia, Medellín, Colombia; ${ }^{2}$ Hospital Universitario San Vicente Fundación, Medellin, Colombia; ${ }^{3} I P S$ Universitaria, Servicios de Salud Universidad de Antioquia, Medellin, Colombia; ${ }^{4}$ Grupo de Epidemiología y Bioestadística Universidad CES, Medellín, Colombia; ${ }^{5}$ Clínica Universitaria Bolivariana, Medellín, Colombia

Background: muscle involvement is frequently reported in polyarteritis nodosa (PAN), mostly as myalgia, muscle swelling, and gait difficulty due to intramuscular arteries involvement, peripheral neuropathy, or myositis with slightly or no elevation of muscle enzymes. Magnetic resonance imaging (MRI) findings of compromised muscles have been reported in isolated cases, mainly as a limited form of PAN, however, muscular involvement patterns in MRI of patients with PAN have been recently described

Objectives: to describe MRI of legs findings in patients with PAN in a tertiary center from Medellin-Colombia.

Methods: it was performed a retrospective cross-sectional descriptive study of 15 adult patients who were clinically assessed as having PAN and who had undergone MRI of legs between January 2011 and December 2019. Characteristics already informed in previous studies, affected structures (muscle, subcutaneous tissue, and bone) and pattern of hyperintensities were described as diffuse pattern (signal alterations affecting the entire area of the involved muscle), patched pattern (areas of hyperintensities alternating areas of normal muscle signal intensity), and fluffy nodular pattern or cotton-wool appearance (round hyperintense lesions with fluffy margins centered on blood vessels).

Results: clinical characteristics: myalgia, especially calf pain, was the most frequent muscular complaint; other clinical manifestations were: constitutional symptoms $(80 \%)$, arthralgias or arthritis $(50 \%)$, mono/polyneuropathy $(33 \%)$, subcutaneous nodules (33\%), livedo reticularis $(20 \%)$, lower limbs ulcers $(13 \%)$, abdominal symptoms (13\%), and purpura $(7 \%)$. MRI findings: bilateral muscular edema was found in all patients (100\%), fatty infiltration (20\%), edema of the subcutaneous cellular tissue $(20 \%)$, and muscular atrophy $(13 \%)$ were also described. A diffuse pattern occurred in $46 \%(n=7)$ of patients (figure panel A), a patched pattern (figure panel B) in $46 \%(n=7)$, and a fluffy nodular pattern or cotton-wool appearance (figure panel C) in $6 \%(n=1)$. The most frequently affected muscular group was gastrocnemius and soleus $(67 \%)$, followed by anterior tibialis (27\%), plantar, long peroneus, first finger flexors, and long flexors only affected in $7 \%$. Bone involvement was found in $53 \%$, being the tibia the most affected, followed by the fibula and the calcaneus. MRI led to guide the site of muscle biopsy to prove histological medium-size vasculitis in half of the patients Conclusion: in patients with PAN suspicion who have muscular complaints, especially calf pain, MRI arises as an important diagnostic tool, and also as a guide to muscular biopsy to prove vasculitis. The patterns associated with PAN are diffuse, patched or fluffy nodular hyperintensities in gastrocnemius and soleus with or without bone compromise.

References:

[1] Kang, Y. et al. Muscle involvement in polyarteritis nodosa: Report of eight cases with characteristic contrast enhancement pattern on MRI. Am. J. Roentgenol. 206, 378-384 (2016).

[2] Hofman DM, Lems WF, Witkamp TD, Putte VD, Bijlsma JW. Demonstration of calf abnormalities by magnetic resonance imaging in polyarteritis nodosa. Clin Rheumatol 1992; 11:402-404.
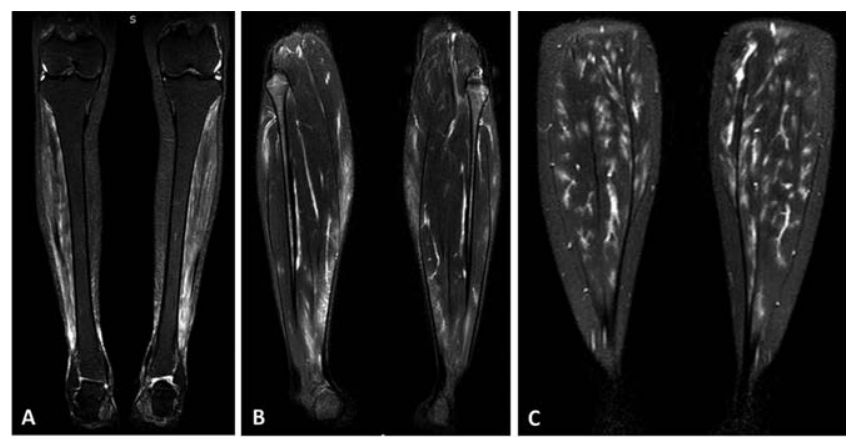

Figure

Disclosure of Interests: None declared

DOI: 10.1136/annrheumdis-2020-eular.6538

\section{FRI0223 SUBPHENOTYPES OF ANCA ASSOCIATED VASCULITIS IDENTIFIED BY LATENT CLASS ANALYSIS}

K. Wóicik ${ }^{1}$, A. Ćmiel ${ }^{2}$, A. Masiak ${ }^{3}$, Z. Zdrojewski ${ }^{3}$, R. Jeleniewicz ${ }^{4}$, M. Majdan ${ }^{4}$ I. Brzosko ${ }^{5}$, M. Brzosko ${ }^{5}$, P. Głuszko ${ }^{6}$, M. Stasiek ${ }^{6}$, M. Wisłowska ${ }^{7}$, J. KurZalewska $^{8}$, M. Madej ${ }^{9}$, A. Hawrot-Kawecka ${ }^{10}$, H. Storoniak ${ }^{3}$, B. Bułto-Piontecka ${ }^{3}$ A. Debska-Ślizien ${ }^{3}$, E. Kucharz ${ }^{10}, K$. Jakuszko ${ }^{9}$, J. Musiał ${ }^{1}$ on behalf of POLVAS. ${ }^{1}$ Jagiellonian University Medical College, Kraków, Poland; ${ }^{2}$ AGH University of Science and Technology, Kraków, Poland; ${ }^{3}$ Medical University of Gdańsk, Gdańsk, Poland; ${ }^{4}$ Medical University of Lublin, Lublin, Poland; ${ }^{5}$ Pomeranian Medical University, Szczecin, Poland; ${ }^{6}$ National Institute of Geriatrics,

Rheumatology and Rehabilitation, Warszawa, Poland; ${ }^{7}$ Central Clinical Hospital of the Ministry of the Interior and Administration, Warszawa, Poland; ${ }^{8}$ Military Institute of Medicine, Warszawa, Poland; ${ }^{9}$ Wroclaw Medical University, Wrocław, Poland; ${ }^{10}$ Medical University of Silesia, Katowice, Poland

Background: ANCA associated vasculitides (AAV) are a heterogeneous group of rare diseases with unknown etiology and the clinical spectrum ranging from life-threatening systemic disease, through single organ involvement to minor isolated skin changes. Thus there is an unmet need for phenotype identification especially among patients with granulomatosis with polyangiitis GPA, patients with microscopic polyangiitis MPA group seems to be more 